\title{
REVIEW
}

\section{Cybernetic principles in the systematic concept of hypothalamic feeding control}

\author{
Oliver Fricke ${ }^{1,2}$, Gerd Lehmkuhl ${ }^{2}$ and Donald W Pfaff ${ }^{1}$ \\ ${ }^{1}$ Laboratory of Neurobiology and Behavior, The Rockefeller University, New York, New York, USA and ${ }^{2}$ Department of Child and Adolescent Psychiatry, \\ University of Cologne, Robert Koch Str, 10 D-50924 Cologne, Germany \\ (Correspondence should be addressed to O Fricke; Email: frickeo@rockefeller.edu)
}

\begin{abstract}
Research on biological mechanisms of eating behavior and related disorders, such as obesity and anorexia nervosa, has become a large field of research in the last 15 years. With the discovery of peptides related to hypothalamic control of food intake (e.g. leptin and ghrelin) the search for the biological 'master key' of feeding control was renewed. As a result, mid-20th century biological concepts based on systematic and cybernetic thoughts fell into oblivion. This review highlights discoveries of hypothalamic-controlled feeding and eating behavior with a cybernetic and systematic perspective. Interestingly, older ideas of hypothalamic function offer possibilities for the incorporation of new molecular discoveries into systematic concepts of feeding behavior.
\end{abstract}

European Journal of Endocrinology 154 167-173

\section{Introduction}

Biological mechanisms of feeding and eating behavior have become an important focus of recent biomedical research due to the high prevalence of diseases related to abnormal feeding behavior. Obesity, metabolic syndrome, and type II diabetes result in cardiovascular diseases and represent the largest part of total mortality in western industrial countries (1). Moreover, anorexia nervosa and bulimia have an increasing prevalence in those countries and their incidence exceeds $1 \%$ in adolescents (2). Understanding the biological mechanisms that organize feelings of hunger and saturation will not necessarily provide the only key to sufficiently treat complex diseases, such as anorexia nervosa, or socio-cultural problems, such as obesity. However, understanding feeding behavior can serve as a first step on a longer and hopefully more successful journey to deal with those diseases and problems for which current medical practice does not provide any convincing and successful treatment. The tremendous success of methods in cell biology and genetics has opened a new chapter for research on hunger and saturation. In 1994, the discovery of leptin began a new era of research on homeostatic functions of the hypothalamus (3), and therefore, older systematic concepts fell into oblivion under the dominance of the discovery of new genes and peptides. This review focuses on those ancient concepts and suggests that a systematic approach might be helpful in understanding recently published data on molecular aspects of the homeostatic control of food intake. This paper is also intended to increase our understanding of how hormones regulate food intake. Hormones are signals and, therefore, are transmitters of information between different players (e.g. controller or controlled variable) in a regulatory loop. In general, cybernetic concepts are not new in endocrinology. Regulation and system theory are the key principles of our discipline, where the signal (hormone) stands at the center of research and treatment.

\section{The concept of regulation}

Norbert Wiener's book Cybernetics, published in 1948, gave birth to a common theory of regulation (4). Wiener chose the name cybernetics from the Greek word kybernos, which is the expression for a Greek navy commander. Essentially, the term regulation can be described as the adaptation of a controlled variable to a given set point in a feedback loop. This process of adaptation is managed by the controller, which records the difference between the controlled variable and the set point (Fig. 1). Therefore, in a mathematical sense, regulation can be described as a problem of variation calculus or of the ergodic theory to find the optimal 


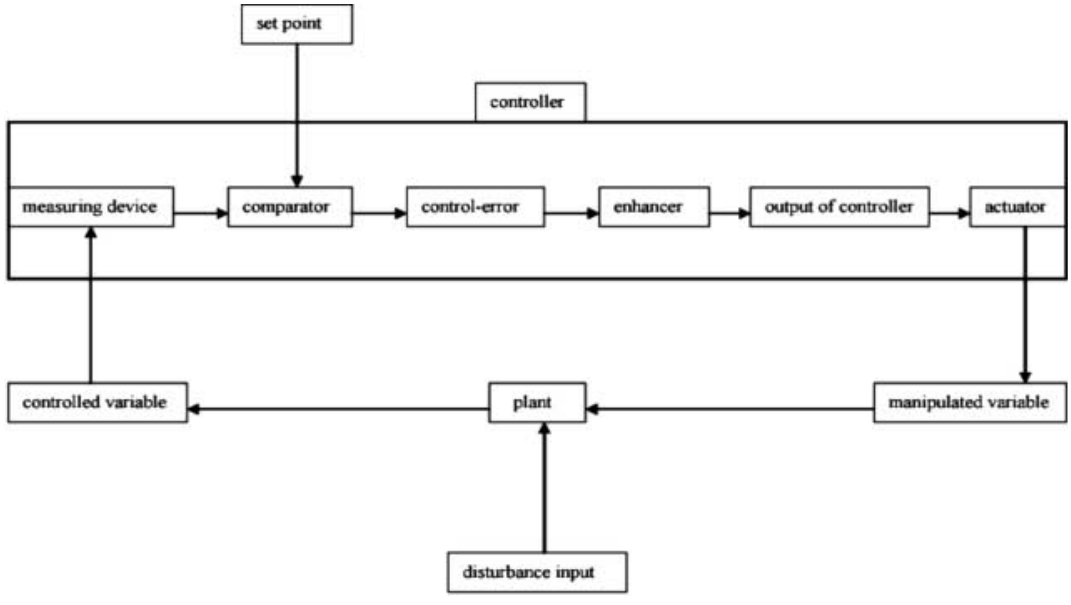

Figure 1 The organization of a feedback loop in a cybernetic system. The controlled variable is compared with the set point. The difference is called control-error and is enhanced by the controller. The enhanced error presents the input to the actuator, which increases or decreases the manipulated variable. The manipulated variable changes the controlled variable, which is again input for the controller. distance between two points in a functional space. The time-related behavior to adapt the controlled variable to the set point might be described by three different scenarios. In the first scenario, the manipulated variable is proportionally changed to the measured difference between the controlled variable and the set point. This kind of regulation is called proportional control and is characterized by the inability to decrease the difference between the controlled variable and the set point to zero. When the response of the controller is dependent on the change of the controlled variable over time, the type of regulation is called differential control. This kind of regulation is more able than the proportional control to hold the controlled variable near the set point as long as the change over time is small in relation to the time constant of the system, which describes the speed of reaction due to changes in the feedback loop. The third type of regulation can be called integral control. The manipulated variable is changed in relation to the sum of changes over time. The advantage of the integral control is that it holds the controlled variable near the set point and is more robust due to larger changes over time than the differential control. The disadvantage of this kind of regulation is that it has a slower reaction to changes in comparison with both the proportional control and differential control mechanisms. Regulation in nature seldom follows one of these three classical mechanisms in a pure way. Rather, most systems can be described by a combination of these three basic scenarios. In this way, advantages of different control strategies are combined. For example, the proportional differential control combines the robustness of the proportional control with the precision of the differential regulation.

The competition for limited resources and, therefore, the optimized adaptation of species to their environmental conditions is the main issue in Darwin's theory of evolution (5). In a teleological sense, the issue of optimization obliges species to develop mechanisms that adapt to environmental conditions. However, this mechanism of adaptation is nothing else than the optimization of a variable in a feedback loop, which can be described by one of the control patterns mentioned above. Therefore, the development of feedback loops might result from powerful selection pressures.

\section{The hypothalamic control of feeding behavior}

The understanding of the mechanisms regulating appetite, hunger and food intake under the prospective of cybernetics requires the interpretation of biological structures as elements of a regulatory system. Classical experiments on the localization of appetite regulating neuronal structures were performed in the middle and second half of the last century. In those experiments, the effect of lesions of hypothalamic areas on food intake and body weight was investigated. Bilateral lesions of the lateral hypothalamic area (LHA) induced aphagic behavior (6). In contrast, lesions of the ventromedial hypothalamus (VMH) resulted in hyperphagic behavior (7).

The dual center hypothesis is based on the results of lesion experiments $(8-10)$. A pioneer in the study of neurobiological mechanisms of food intake, Elliot Stellar, localized the generation of appetite in the LHA and the feeling of saturation in the VMH. In this context, saturation means the lack of any drive to consume food to accomplish a feeling of pleasure. Later experimental results could not support this theory and cast the basic foundations of the dual center hypothesis in a different light. Lesions of the VMH increased the parasympathic activity and, therefore, augmented insulin secretion (11). Elevated insulin secretion induced hyperphagia and increased lipogenesis. These symptoms of VMH lesions disappeared when a vagotomy was performed (12). Lesions of the LHA stood in physiological contrast to $\mathrm{VMH}$ lesions. Bilateral lesions of the vagal nerve decreased parasympathic activity and therefore vagal activity. Therefore, insulin secretion was decreased and the duration of digestion was increased, resulting in a longer feeling of saturation after food intake (13). Moreover, lesions of the medial 
forebrain bundle (MFB), which is anatomically localized near the LHA, resulted in a neglect of appetite-inducing sensory stimuli $(14,15)$. Those dopaminergic fibers transmit the general sensitivity to sensory stimuli (arousal). Therefore, lesions of the MFB reduce drivedependent behavior that also includes feeding behavior.

The discovery of peripheral feedback signals such as leptin $(3,16)$ and ghrelin $(17,18)$ allowed the discovery of hypothalamic and cortical cell populations that are involved in the regulation of food intake. The arcuate nucleus (ARC) represents a main target of leptin binding, and lesions of the lateral area of the LHA result in hyperphagic behavior because anorexigenic neurons are laterally localized (19). The paraventricular and dorsomedial nuclei (PVN, DMN) of the hypothalamus are areas where cell populations with appetite inhibiting characteristics are localized (19, 20). The distributions of some of the hypothalmic populations involved in feeding control are shown in Fig. 2.

In addition to hypothalamic cell populations, there exist neuronal structures with an influence on feeding behavior. Vagal afferents transmit information about intramural gastric pressure, the composition of meals (lipids, glucose, amino acids) and food temperature $(10,21-23)$. These afferents are in synaptic contact with neurons of the nucleus tractus solitarius (NTS), which project to the LHA, PVN and ARC (19, 24). Moreover, the peptide hormone cholecystokinin (CCK) possesses appetite-reducing characteristics in interaction with neurons of the NTS (25). Obviously, vegetative signals related to digestive activity are processed by neuronal cell populations localized in the NTS. Interestingly, estradiol modulates these by binding to estrogen receptor alpha (26). Thereby, estradiol decreases the amount of ingested food per meal, but does not affect the number of meals.

\section{Estrogens and monoamines modulate food intake}

Intracerebroventricularly administered estrogens inhibit food intake. Moreover, cerebral infusion of androgens also has an inhibitory effect on food intake, but only after aromatization to estrogen (27). In contrast, the systemic application of androgens

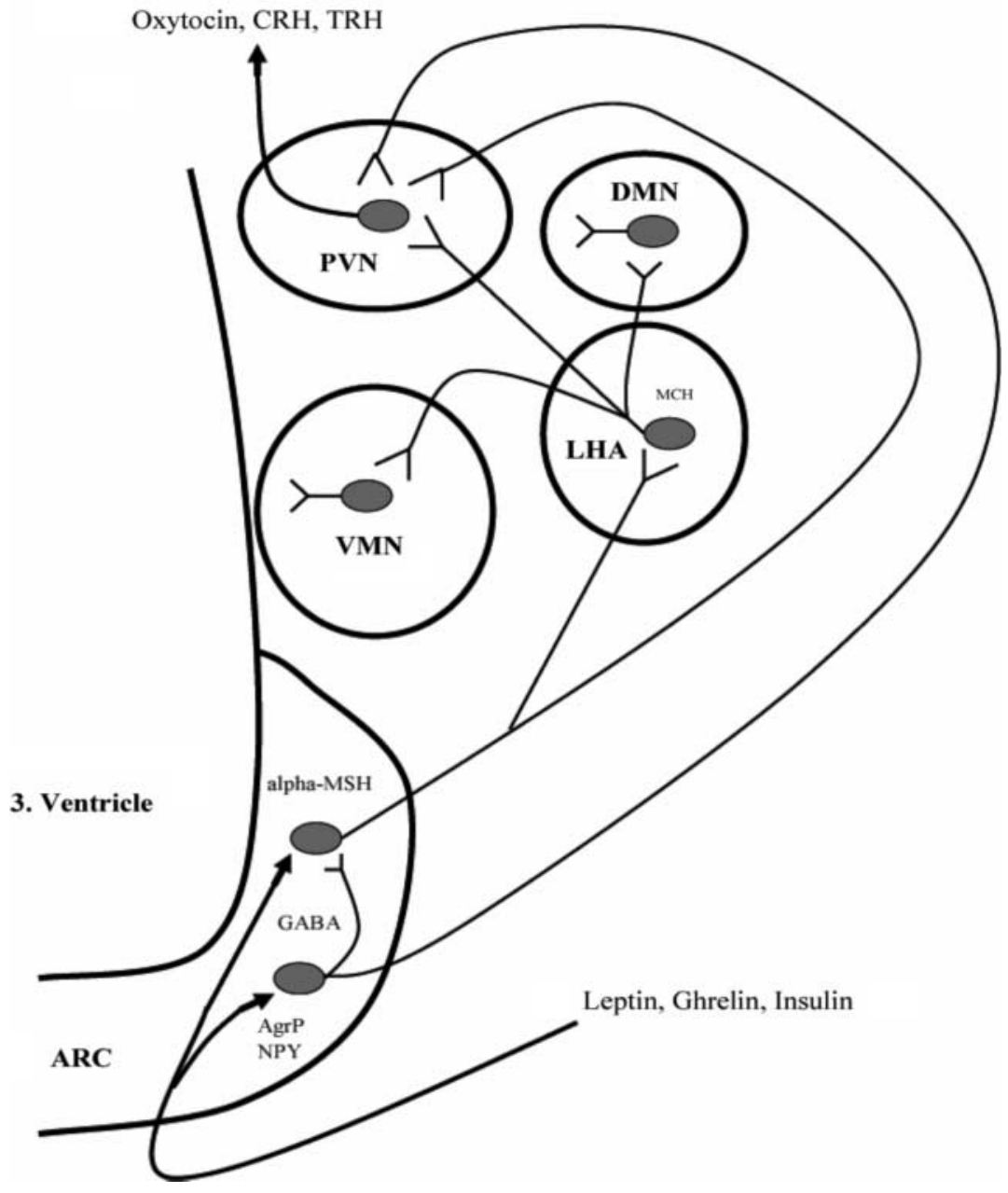

Figure 2 Hypothalamic anatomy involved in feeding control. The illustration sketches the main connections known among hypothalamic cell populations controlling food intake, in a manner that emphasizes the cybernetic construction of the neuronal network. Therefore, additional pathways (e.g. connections between ARC and VMN, neuronal leptin receptors in the VMN) are left out to preserve clarity. The primary feedbacks of leptin, ghrelin and insulin are localized in orexigenic neurons (co-expressing Agouti-related protein (AgrP), NPY and gamma-amino butyric acid (GABA)) and anorexigenic neurons (expressing alpha$\mathrm{MSH}$, deriving from POMC) of the ARC projecting to the PVN and LHA. Moreover, orexigenic neurons (expressing $\mathrm{MCH}$ ) of the LHA project to anorexigenic neurons of the VMN and DMN. Interestingly, secretory cells of the PVN release food inhibiting hormones (corticotropin-releasing hormone (CRH), thyrotropin-releasing hormone (TRH) and oxytocin). 
mainly induces anabolic effects transmitted by skeletal muscles (28). Because many hypothalamic neurons possess a high number of binding sites for estrogens, the modulation of feeding behavior by a direct mechanism on hypothalamic neurons can be suggested (29). This was emphasized by the discovery that estrogens decrease melanin-concentrating hormone (MCH) expression in orexigenic neurons of the LHA $(30,31)$. Moreover, estrogens modify electrical properties of proopiomelanocortin (POMC)-expressing anorexigenic neurons by a recently discovered $\mathrm{G}$ protein-coupled membrane receptor, which activates phospholipase $\mathrm{C}$ (PLC) and likely increases the cytoplasmic calcium concentration $(32,33)$. Thus, intracellular calcium often serves as a second messenger to connect signals by $\mathrm{G}$ protein-coupled membrane receptors with ion channel function. Oscillations of cytoplasmic calcium are present in neurons of the ARC. Thus, these oscillations are dependent on the presence of extracellular calcium (Fig. 3). Interestingly, 17 $\beta$-estradiol (10 nmol/l) induces the release of intracellular calcium in neurons of the ARC characterized by calcium oscillations (Fig. 3). Therefore, estrogens might modify electrical properties of POMC-expressing anorexigenic neurons by the release of intracellular calcium in a cascade involving PLC and calcium-dependent protein kinases.

The monoamines, noradrenaline, dopamine and 5-hydroxytryptamine (5-HT), also possess modulating effects on food intake (19). The injection of noradrenaline into the PVN enhanced food intake in rodents (34). The physiological relevance of this result is emphasized

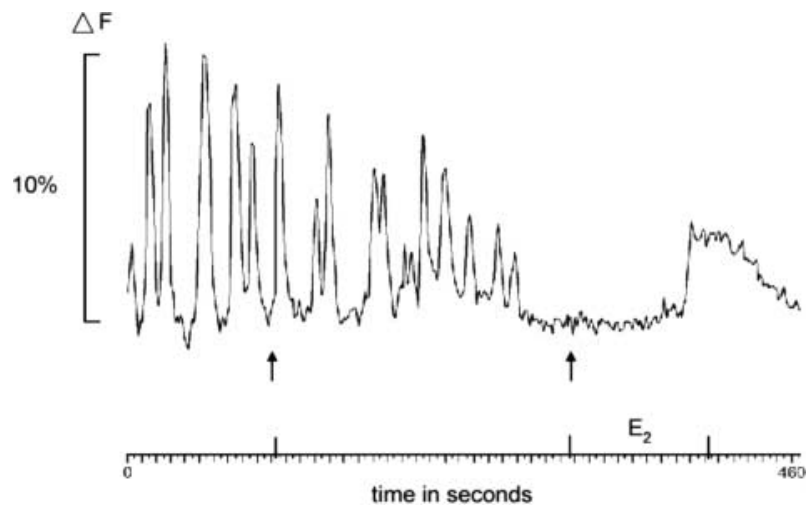

Figure 3 Estradiol releases intracellular calcium in hypothalamic neurons that are characterized by calcium oscillations. The figure displays the recording of changes of free cytoplasmic calcium (related to $\Delta \mathrm{F}$ ) over time (in seconds) in a hypothalamic (ARC) neuron. The recording was performed in a mouse brain slice (ovariectomized female mouse) of $200 \mu \mathrm{m}$ thickness by ratiometric calcium-imaging (fura-2 AM). The neuron shows calcium oscillations under physiological conditions (regular artificial cerebrospinal fluid (R-ACSF) perfusion). When extracellular calcium is removed (first arrow), the oscillations disappear. When 17 $\beta$ estradiol ( $10 \mathrm{nmol} / / \mathrm{E}_{2}$ ) is added (second arrow), a significant calcium transient is visible. Therefore, $\mathrm{E}_{2}$ triggers intracellular calcium release in hypothalamic neurons within seconds (Fricke $\mathrm{O}$ and Pfaff DW, unpublished data). by the discovery of noradrenergic afferents from the locus coeruleus to the PVN (35). As mentioned above, the destruction of dopaminergic afferents of the MFB from the tegmental area results in a neglect of appetite-inducing sensory stimuli. In contrast, dopamine can inhibit food intake, decreasing neuropeptide $\mathrm{Y}$ (NPY) levels in neurons of the ARC projecting to the PVN (36). Furthermore, 5-HT inhibits feeding behavior, which might depend upon projections from neurons of the mesencephalic raphe nuclei to hypothalamic neurons (37). Interestingly, 5-HT-containing neurons of the raphe nuclei may have estrogen receptors, which could transmit estrogen effects on feeding behavior over serotoninergic projections (38). Also, alpha-melanocytestimulating hormone ( $\alpha \mathrm{MSH})$ - containing neurons of the hypothalamic neuronal network have 5-HT2c receptors, which could be targets of serotoninergic afferents (39). Surprisingly, a detailed physiological model as to how 5-HT inhibits feeding behavior does not exist. Nevertheless, serotonin reuptake inhibitors have recently be used for the pharmacological treatment of obesity (40).

In summary, hypothalamic areas receive information on the metabolic conditions of the organism in different quality (e.g. meal size or meal content), which also stand in relation to body mass. Serum leptin levels have a close relationship with body fat mass. Therefore, decreasing leptin serum levels indicate a reduction in body fat mass. In contrast, increasing leptin levels characterize an augmentation of the body fat content (41). The described linear relationship between leptin and body weight is altered in females affected with anorexia nervosa. In contrast to weight loss, these females show higher leptin serum levels at the same body weight when they gain weight, which is a significant risk for the occurrence of relapses $(42,43)$. Ghrelin and CCK are gastrointestinal hormones that provide feedback signals of the gastrointestinal activity to the central nervous system. Because the release of CCK is also dependent on the nutritional fat content, gastrointestinal hormones also transmit information on the composition of ingested nutrition. Moreover, insulin serum levels are related to carbohydrate intake and metabolism and therefore report information on anabolic or catabolic conditions of peripheral tissues (e.g. adipose tissue and skeletal muscle) to hypothalamic neurons (44). Increasing insulin levels indicate a switch to anabolism, whereas falling insulin levels characterize a change to catabolic conditions. Finally, parasympathic vagal afferents transport information on the metabolic body condition, which was also found for the gastrointestinal system (25).

Hormones and neuronal signals might function as controlled variables in a cybernetic model. Hypothalamic neurons with binding sites of metabolic feedback signals and with contact to vagal afferents could function as sensors. Obviously, estrogens and monoaminergic afferents modulate the sensor sensitivity and integrate 
different aspects of homeostasis (e.g. thermogenesis and reproductive behavior) in the context of sensory stimuli and responses. This issue can be summarized under the concept of the nterplay of general arousal with different modules of behavior (e.g. feeding or mating behavior, nursing behavior) (45). Rodents have an increased response to sexual stimuli after priming with estrogens, whereas nutritional stimuli are neglected (46).

The feedback signal is compared with the set point and the resulting control error is sent to the actuator after enhancement. Under consideration of the present experimental data, estrogens and monoaminergic afferents cannot provide the function of enhancement on feedback signals of food intake, because in this case the control error is enhanced independently of the direction of the error (too much or too little energy storage). Thus, the directional effects, enhancement or inhibition, on feeding behavior cannot be explained by estrogens and monoamines. More likely, estrogens and monoamines change the set point of the controller. In this way, a directional effect on feeding behavior becomes explicable, because the feedback loop drifts to less or more food intake when estrogens or monoamines are administered.

\section{Cybernetics of body weight regulation}

In a cybernetic model, hormones like leptin, ghrelin and insulin represent controlled variables that transmit the feedback of the manipulated variables: body mass, body composition, digestion, metabolism and thermogenesis. As mentioned above, vagotomy can partly inhibit the effect of hypothalamic lesions on feeding behavior. Therefore, signals of the actuator are partly transmitted by parasympathic efferents, which are under the control of the LHA and VMN. In conclusion, the hypothalamus represents a sensor and functions partly as the actuator in a control system of feeding behavior. Hormones (e.g. estrogens) and neuronal afferents (monoaminergic system) modulate food intake by influencing the set points of controlled variables. The mode of control is also an interesting issue in the discussion about the regulation of food intake. It is likely that rapid physiological controls (e.g. the glucose serum level, in minutes) are mediated using the mode of differential controls. In contrast, the regulation of body fat mass needs a robust and more noise-insensitive control, because body weight is adjusted over longer time periods. Therefore, proportional or integral control provides an advantage in the regulation of body weight.

Recently, Pinto et al. showed that removing and replacing leptin changes the synaptic contact and input to orexigenic and anorexigenic neurons (containing NPY and POMC respectively) in ARC (47). Removing the leptin signal increased the synaptic density of inihibitory neurons to anorexigenic neurons, whereas the synaptic density and input were decreased for the contact with excitatory neurons. On orexigenic neurons, however, the result was the opposite. The replacement of leptin recovered the effect. Because leptin can be considered to be a feedback signal of body fat content, and neurons of ARC can be considered to be the main target of the leptin signal, this neuronal circuitry resembles a regulatory working control element. Excitatory and inhibitory afferents serve as a contrast enhancement in the measurement of leptin change. Hypothetically, the binding of leptin changes the neuronal firing frequency. The subsequent change of synaptic input alternates the firing rate. This contrast enhancement reduces the signal-to-noise ratio in the circuitry of energy control. Moreover, the efferent output of the first neuron in the neuronal network affects the firing frequency of the upstream neuron over synaptic activation. In this way, differential responses to leptin changes are integrated by the second neuron in this hierarchically constructed network, which results in a proportional response of the firing frequency to leptin changes. In conclusion, recently published results elucidate the physiological process of homeostatic control of body weight at a molecular level. Interestingly, these novel results on neuronal networks in the hypothalamus have revitalized ancient concepts of the regulation of food intake and call for a more systematic approach to the biological concepts of feeding behavior (Fig. 4).

The description of physiological processes using cybernetic models attributes variables of a control system to biological structures, whereas manipulated and controlled variables are connected to each other by a transfer function. The attribution of control elements to biological structures always contains the problem of semantic simplification, because the biological structure is reduced to a singular meaning. The same biological structure can be a controlled variable in a certain feedback loop and a manipulated variable in a different loop. Therefore, feedback loops might be organized in chains, where elements are parts of different loops in different functions. The present data about the effect of estrogens and monoamines on feeding and mating behavior suggest the possible presence of chained loops to form higher order control systems, metamodels of regulation, for example the chained control of food intake with sexual behavior as briefly mentioned above.

In conclusion, the interpretation of recent data on feeding behavior from a systematic point of view, provides a strategy that might help increase our understanding of hormonal effects on eating disorders. Thus, older concepts may serve as a useful basis for the interpretation of novel discoveries about the neurobiological mediation of hunger.

\section{Acknowledgements}

O F thanks the Max-Kade Foundation for support of his work. Moreover, we thank Dr Aaron M Jasnow for a fruitful discussion. 


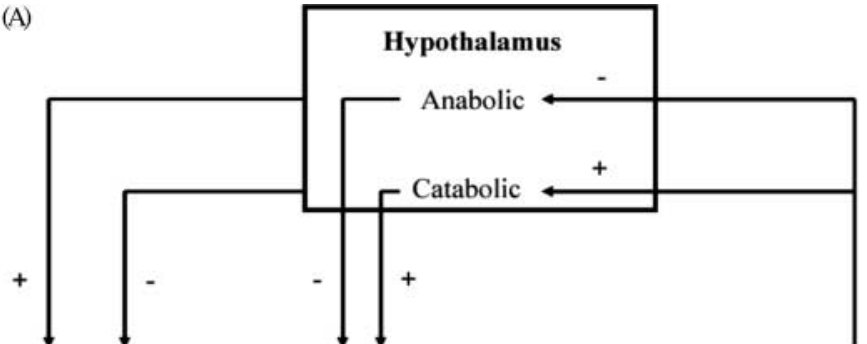

Food intake

Energy expenditure
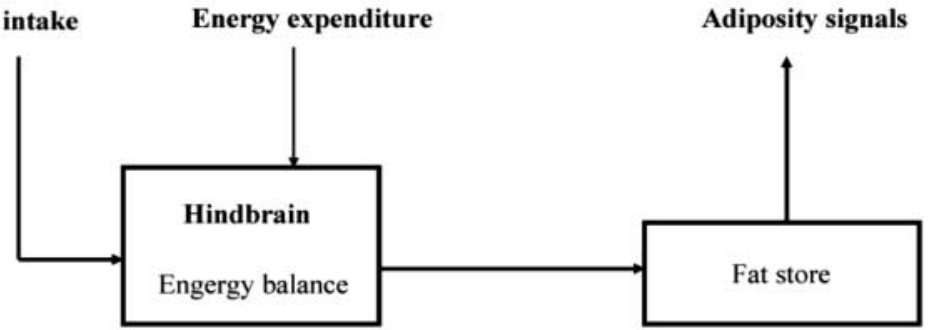

(B)

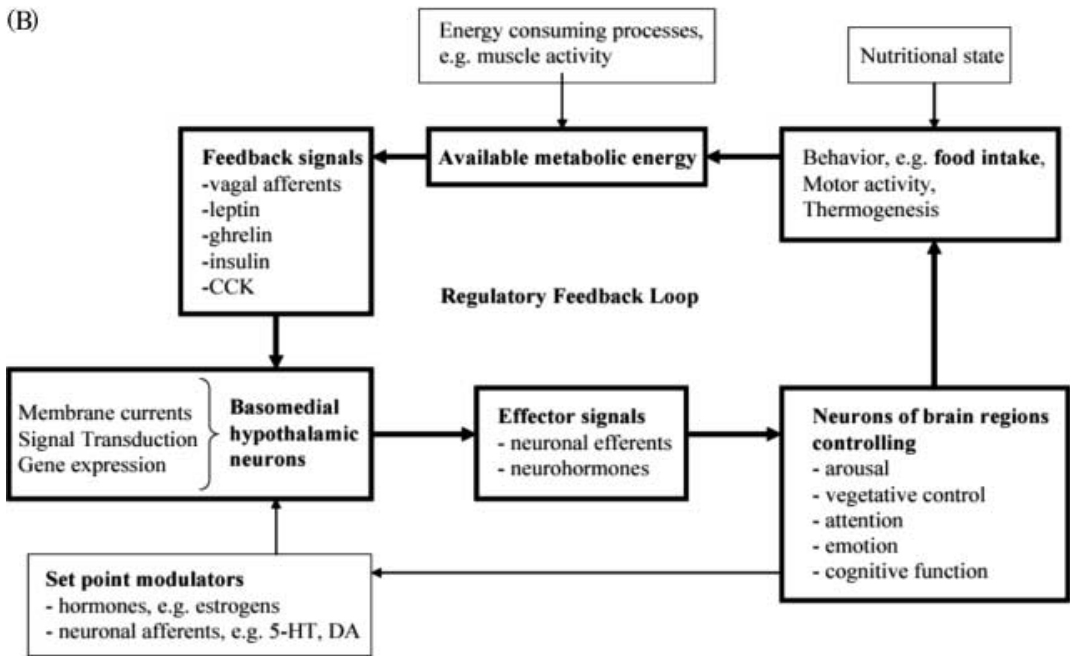

Figure 4 A systematic approach to feeding and eating behavior. (A) Model for the control of feeding behavior and energy expenditure adapted from Schwartz et al. (19). The reorganization of this model under a cybernetic approach introduces a set point (B). The set point is modified by hormones, e.g. estradiol, or neuronal afferents, e.g. serotoninergic or dopaminergic (DA) afferents to basomedial hypothalamic neurons. Feedback neurons integrate homeostatic and sensory information and send efferents to neuronal networks organizing behavior and homeostasis (actuator networks). Understanding why different homeostatic and environmental conditions lead to different stable states of energy storage and energy expenditure is enabled by this cybernetic approach.

\section{References}

1 Bell CG, Walley AJ \& Froguel P. The genetics of human obesity. Nature Reviews Genetics 20056 221-234.

2 Cotrufo P, Gnisci A Caputo I \& Brief report, Psychological characteristics of less-severe forms of eating disorders: an epidemiological study among 259 female adolescents. Journal of Adolescence $200528147-154$.

3 Zhang Y, Proenca R, Maffei M, Barone M, Leopold L \& Friedman JM. Positional cloning of the mouse obese gene and its human homologue. Nature 1994372 425-432.

4 Wiener N. Cybernetics or Control and Communication in the Animal and the Machine. Cambridge, USA: MIT Press, 1948.

5 Darwin C. On the Origin of Species by Means of Natural Selection. London: John Murray, 1859.

6 Anand BK \& Brobeck JR. Hypothalamic control of food intake in rats and cats. Yale Journal of Biology and Medicine 195124 123-140.

7 Duggan JP \& Booth DA. Obesity, overeating and rapid gastric emptying in rats with ventromedial hypothalamic lesions. Science $1986231609-611$.

8 Stellar E. The physiology of motivation. Psychology Reviews 1954 $615-22$.
9 Heterington AW \& Ranson SW. Hypothalamic lesions and adiposity in the rat. Anatomical Record $1940 \mathbf{7 8} 149-172$.

10 Anand BK \& Brobeck JR. Localization of a 'feeding center' in the hypothalamus of the rat. Proceedings of the Society for Experimental Biology and Medicine 195177 323-324.

11 Steffens AB. Plasma insulin content in relation to blood glucose level and meal pattern in the normal and hypothalamic hyperphagic rat. Physiology and Behavior 1970 5 147-151.

12 Inoue S, Bray GA \& Mullen YS. Transplantation of pancreatic beta-cells prevents development of hypothalamic obesity in rats. American Journal of Physiology 1978235 266-271.

13 Powley TL \& Keesey RE. Relationship of body weight to the lateral hypothalamic feeding syndrome. Journal of Comparative and Physiological Psychology 197070 25-36.

14 Ungerstedt U. Adipsia and aphagia after 6-hydroxydopamine induced degeneration of the nigro-striatal dopamine system. Acta Physiologica Scandinavica 1971367 (Suppl) 95-122.

15 Marshall JF, Turner BH \& Teitelbaum P. Sensory neglect produced by lateral hypothalamic damage. Science $1971 \mathbf{1 7 4} 523-525$.

16 Friedman JM \& Halaas JL. Leptin and the regulation of body weight in mammals. Nature 1998395 760-763.

17 Tschop M, Smiley DL \& Heiman ML. Ghrelin induces adiposity in rodents. Nature $2000 \mathbf{4 0 7} 908-913$. 
18 Horvath TL, Diano S, Sotonyi P, Heiman M \& Tschop M. Minireview: ghrelin and the regulation of energy balance - a hypothalamic perspective. Endocrinology 2001142 4163-4169.

19 Schwartz MW, Woods SC, Porte D Jr, Seeley RJ \& Baskin DG. Central nervous system control of food intake. Nature $2000 \mathbf{4 0 4}$ $661-671$.

20 Bellinger LL \& Bernardis LL. The dorsomedial hypothalamic nucleus and its role in ingestive behavior and body weight regulation: lessons learned from lesioning studies. Physiology and Behavior $200276431-442$.

21 Mei N. Intestinal chemosensitivity. Physiological Reviews 198565 211-237.

22 Schwartz GJ. The role of gastrointestinal vagal afferents in the control of food intake: current prospects. Nutrition $2000 \mathbf{1 6}$ $866-873$.

23 Strader AD \& Woods SC. Gastrointestinal hormones and food intake. Gastroenterology 2005128 866-873.

24 Ter Horst GJ, de Boer P, Luiten PG \& van Willigen JD. Ascending projections from the solitary tract nucleus to the hypothalamus. A Phaseolus vulgaris lectin tracing study in the rat. Neuroscience $198931785-797$.

25 Geary N. Estradiol and appetite. Appetite 200035 273-274.

26 Geary N, Asarian L, Korach KS, Pfaff DW \& Ogawa S. Deficits in $\mathrm{E}_{2}$-dependent control of feeding, weight gain and cholecystokinin satiation in ER-alpha null mice. Endocrinology $2001 \mathbf{1 4 2}$ 4751-4754.

27 Nunez AA, Gray JM \& Wade GN. Food intake and adipose tissue lipoprotein lipase activity after hypothalamic estradiol benzoate implants in rats. Physiology and Behavior 198025 595-598.

28 Rand MN \& Breedlove SM. Androgen locally regulates rat bulbucavernosus and levator ani size. Journal of Neurobiology 199223 $17-30$.

29 Krieger MS, Morrell JI \& Pfaff DW. Autoradiographic localization of estradiol-concentrating cells in the female hamster brain. Neuroendocrinology 197622 193-205.

30 Mystkowski P, Seeley RJ, Hahn TM, Baskin DG, Havel PJ, Matsumoto AM, Wilkinson CW, Peacock-Kinzig K, Blake KA \& Schwartz MW. Hypothalamic melanin-concentrating hormone and estrogen-induced weight loss. Journal of Neuroscience 2000 $208637-8642$.

31 Morton GJ, Mystkowski P, Matsumoto AM \& Schwartz MW. Increased hypothalamic melanin concentrating hormone gene expression during energy restriction involves a melanocortinindependent, estrogen-sensitive mechanism. Peptides $2004 \mathbf{2 5}$ $667-674$.

32 Qiu J, Bosch MA, Tobias SC, Grandy DK, Scanlan TS, Ronnekleiv OK \& Kelly MJ. Rapid signaling of estrogen in hypothalamic neurons involves a novel G-protein-coupled estrogen receptor that activates protein kinase C. Journal of Neuroscience 200323 9529-9540.

33 Kelly MJ, Qiu J \& Ronnekleiv OK. Estrogen modulation of G-protein-coupled receptor activation of potassium channels in the central nervous system. Annals of the New York Academy of Sciences $2003 \mathbf{1 0 0 7} 6-16$.

34 Leibowitz S, Roossin P \& Rosenn M. Chronic norepinephrine injection into the hypothalamic paraventricular nucleus produces hyperphagia and increased body weight in the rat. Pharmacology, Biochemistry and Behavior 198421 801-808.

35 Wellman PJ. Norepinephrine and the control of food intake. Nutrition $200016837-842$.

36 Bina KG \& Cincotta AH. Dopaminergic agonists normalize elevated hypothalamic neuropeptide $\mathrm{Y}$ and corticotropin-releasing hormone, body weight gain, and hyperglycemia in ob/ob mice. Neuroendocrinology $2000 \mathbf{7 1} 68-78$.

37 Leibowitz S \& Alexander J. Hypothalamic serotonin in control of eating behavior, meal size and body weight. Biological Psychiatry $199844851-862$.

38 Alves SE, McEwen BS, Hayashi S, Korach KS, Pfaff DW \& Ogawa S. Estrogen-regulated progestin receptors are found in the midbrain raphe but not hippocampus of estrogen receptor alpha (ERalpha) gene-disrupted mice. Journal of Comparative Neurology $2000 \mathbf{4 2 7}$ $185-195$.

39 Heisler LK, Cowley MA, Tecott LH, Fan W, Low MJ, Smart JL, Rubinstein M, Tatro JB, Marcus JN, Holstege H, Lee CE, Cone RD \& Elmquist JK. Activation of central melanocortin pathways by fenfluramine. Science 2002297 609-611.

40 Ljung T, Ahlberg AC, Holm G, Friberg P, Andersson B, Eriksson E \& Bjorntorp P. Treatment of abdominally obese men with a serotonin reuptake inhibitor: a pilot study. Journal of Internal Medicine 2001250 219-224.

41 Kalra SP, Dube MG, Pu S, Xu B, Horvarth TL \& Kalra PS. Interacting appetite-regulating pathways in the hypothalamic regulation of body weight. Endocrine Reviews 199920 68-100.

42 Mantzoros C, Flier JS, Lesem MD, Brewerton TD \& Jimerson DC. Cerebrospinal fluid leptin in anorexia nervosa: correlation with nutritional status and potential role in resistance to weight gain. Journal of Clinical Endocrinology and Metabolism 199782 $1845-1851$.

43 Holtkamp K, Hebebrand J, Mika C, Heer M, Heussen N \& HerpertzDahlmann B. High serum leptin levels subsequent to weight gain predict renewed weight loss in patients with anorexia nervosa. Psychoneuroendocrinology 200429 791-797.

44 Bruning JC, Gautam D, Burks DJ, Gillette J, Schubert M, Orban PC, Klein R, Krone W, Muller-Wieland D \& Kahn CR. Role of brain insulin receptor in control of body weight and reproduction. Science $20002892122-2125$.

45 Mong JA \& Pfaff DW. Hormonal and genetic influences underlying arousal as it drives sex and aggression in animal and human brains. Neurobiology of Aging 200324 (Suppl 1) S83-S88.

46 Mong J, Easton A, Kow LM \& Pfaff D. Neural, hormonal and genetic mechanisms for the activation of brain and behavior. European Journal of Pharmacology $2003480229-231$.

47 Pinto S, Roseberry AG, Liu H, Diano S, Shanabrough M, Cai X, Friedman JM \& Horvath TL. Rapid rewiring of arcuate nucleus feeding circuits by leptin. Science $2004 \mathbf{3 0 4} 110-115$.

Received 11 May 2005

Accepted 28 October 2005 